\title{
Zur bewusstseinsanalytischen Philosophie von Walther Schmied-Kowarzik
}

\author{
Wolfdietrich Schmied-Kowarzik \\ Institut für Philosophie, Universität Kassel
}

Durch die Vermittlung des finnischen Philosophen Wilhelm Bolin wird Walther Schmied-Kowarzik (1885-1958) aus Wien/Österreich als erster Ordinarius für Philosophie an die 1919 neu gegründete estnische Landesuniversität Tartu berufen. Er sollte dort nicht nur die Fachgebiete Philosophie, Psychologie und Pädagogik nach dem Vorbild westeuropäischer Universitäten aufbauen, sondern auch dafür sorgen, dass innerhalb von zehn Jahren diese Fächer in estnischer Sprache zu fundamentalen Bausteinen der estnischen Lehrerbildung und akademischen Kultur werden können. Walther Schmied-Kowarzik vertrat eine bewusstseinsanalytische (phänomenologische) Philosophierichtung, wie sie zu Beginn des 20. Jahrhunderts aufkommt und bis heute fortbesteht. Schmied-Kowarzik geht es dabei um eine transzendentale Analyse der Formen des „subjektiven, “des „objektiven“ und des „ideellen Geistes“. Die subjektiven Formen menschlichen Erkennens, Fühlens und Wollens in reflexiver Rückwendung zu differenzieren hatte Schmied-Kowarzik in seiner Wiener Habilitationsschrift Umriß einer analytischen Psychologie (1912/1928) grundgelegt. Hierauf aufbauend, entwickelt er in seiner in Tartu erschienenen Schrift Die Objektivation des Geistigen. Der objektive Geist und seine Formen (1927) die Grundrisse einer Kulturphilosophie. Nach seiner Wegberufung an die Pädagogische Akademie in Frankfurt am Main 1927 wendet er sich sodann in seiner Ethik. Mit Berücksichtigung pädagogischer Probleme (1932) den Formen des „ideellen Geistes“ zu, dem auch seine letzte, posthum erschienene Schrift Frühe Sinnbilder des Kosmos. Gotteserlebnis und Welterkenntnis in der Mythologie (1974) gewidmet ist.

Schlagwörter: Walther Schmied-Kowarzik, die Formen des subjektiven Geistes, Bewusstseinsanalytische Philosophie (Phänomenologie), Kultur als Objektivation des Geistigen, Sitte und Religion als Formen des ideellen Geistes

Korrespondenzanschrift: Wolfdietrich Schmied-Kowarzik, Universität Kassel, Institut für Philosophie, Nora-Platiel-Str. 1,34127 Kassel, Deutschland.E-Mail: schmiedk@uni-kassel.de.

An abstract in English is available at the end of the article. 


\section{Zur Berufung und zum Wirken von Walther Schmied- Kowarzik in Tartu}

Nach dem Zusammenbruch des zaristischen Russlands und den folgenden Kriegswirren mussten in der 1918 neu gegründeten Republik Estland erst die Grundlagen für die Errichtung und den Erhalt der estnischen Kultur geschaffen werden. Die Einführung des Estnischen als Landessprache machte eine Befreiung des gesamten Bildungswesens aus der bis dahin vorherrschenden Dominanz der deutschen und der russischen Sprache und Kultur erforderlich. In diesem Sinne galt es 1919 auch die vormals deutsch- und russischsprachige Universität Dorpat schrittweise als estnische Landesuniversität Tartu völlig neu aufzubauen.

Aber in den ersten Jahren gab es noch nicht genügend estnische Akademiker, die die ganze Breite der universitären Disziplinen ausfüllen konnten. Also holte man sich vor allen bei den sprachverwandten Finnen Hilfe und Verstärkung, doch auch diese reichte nicht aus. Die Berufung von Ungarn - ebenfalls zur finno-ugrischen Sprachfamilie gehörig — brachte nicht den erwünschten leichteren Einstieg, denn die beiden Sprachlinien des FinnoUgrischen waren geschichtlich zu lange getrennt, so dass keine direkte Verständigung möglich war. Deshalb wurden in einer weiteren Berufungsrunde doch wieder deutschsprachige Wissenschaftler berufen, die in einer auf 10 Jahre befristeten Übergangszeit eine neue estnischsprachige Wissenschaftlergeneration heranbilden sollten.

Von Estland aus um seinen Rat gefragt, schlug der einflussreiche finnisch-schwedische Philosoph Wilhelm Bolin, Direktor der Universitätsbibliothek von Helsinki, den jungen österreichischen Philosophen Walther Schmied-Kowarzik vor, der dann 1920 primo et unico loco auf das Ordinariat für Philosophie der estnischen Landesuniversität Tartu berufen wurde. Wilhelm Bolin (1835-1924) war durch die gemeinsame Herausgabe der Werke von Ludwig Feuerbach (1903-1910) eng mit dem Wiener Ordinarius Friedrich Jodl befreundet, der 1914 verstorben war. ${ }^{1}$ Und einer der engsten Schüler Friedrich Jodls (1849-1914) war Walther Schmied-Kowarzik, er wurde von Jodl 1908 promoviert und habilitierte sich 1913 an der Philosophischen Fakultät der Wiener Universität. Nach dem Tode von Friedrich Jodl finanzierte Wilhelm Bolin die Herausgabe der nachgelassen Schriften von Jodl und zum Kreis der Herausgeber um die Witwe Margarete Jodl gehörte auch Walther Schmied-Kowarzik. ${ }^{2}$ So lag es nahe, dass Wilhelm Bolin Schmied-Kowarzik für die Berufung an die neu gegründete Universität Tartu vorschlug.

Walther Schmied-Kowarzik ${ }^{3}$ hatte von 1904 bis 1908 Philosophie, Ge-

\footnotetext{
$1 \mathrm{Zu}$ Bolin und Jodl siehe (Gimpl 1990) sowie (Gimpl 1996).

${ }^{2}$ Siehe beispielsweise (Jodl 1920) sowie (Jodl 1924).

3 Geb. am 22. Mai 1885 in Mödling bei Wien (Österreich), gest. am 24. Juli 1958 in Mödling.
} 
schichtswissenschaft und Germanistik bei Friedrich Jodl, Alois Höfler und Richard Reininger an der Universität Wien studiert (mit einem Studiensemester 1906/07 in Berlin bei Wilhelm Dilthey und Alois Riehl). 1908 wurde er mit der grundlegenden Arbeit Zeit und Raum (1908) von Jodl promoviert und nach einigen weiteren Studien (Schmied-Kowarzik 1910 sowie Schmied-Kowarzik 1911) legte Walther Schmied-Kowarzik 1912 das umfangreiche Buch Umriß einer neuen analytischen Psychologie und ihr Verhältnis zur empirischen Psychologie (1912) ${ }^{4}$ vor, mit dem er sich 1913 auf Vorschlag von Friedrich Jodl in der Philosophischen Fakultät der Universität Wien habilitierte.

Unbestreitbar war Walther Schmied-Kowarzik einer der intimsten Schüler Friedrich Jodls und stark beeinflusst von Jodls anthropologischer Kulturphilosophie und Ethik (vgl. Schmied-Kowarzik 1996). Aber ebenso unbestreitbar stand er dem bewusstseinsanalytischen Ansatz von Alois Höfler (1853-1922) nahe und war in diesem Sinne ein Enkelschüler des Wiener Philosophen Franz Brentano (1838-1917). Von hierher ist auch die Nähe zu Edmund Husserls (1859-1938) Phänomenologie erklärlich, die er in seinen späteren Schriften auch ausdrücklich thematisiert hat. Friedrich Jodl war souverän genug, diese Hinwendung seines Schülers zu der von ihm abgelehnten bewusstseinsanalytischen Philosophie, nicht nur zu tolerieren, sondern sogar verständnisvoll zu begleiten (vgl. Schmied-Kowarzik 1993). Der schroffe Gegensatz zwischen der bewusstseinsanalytischen Philosophie Franz Brentanos und dem wissenschaftsorientierten Realismus Friedrich Jodls wurde von Schmied-Kowarzik über die geisteswissenschaftliche Psychologie und hermeneutische Kulturphilosophie von Wilhelm Dilthey (1833-1911) vermittelt, dem dritten großen Lehrer, dem sich Walther Schmied-Kowarzik in seinem eigenen philosophischen Schaffen verpflichtet fühlte.

Nach einem Gastsemester an der Hochschule in Göteborg/Schweden im Sommer 1920 nimmt Walther Schmied-Kowarzik den Ruf auf das Ordinariat für Philosophie an der neu gegründeten estnischen Landesuniversität Tartu an. Seine Aufgabe war von Anfang an eine doppelte, zum einen sollte er das philosophische Seminar und seine Unterabteilungen der Psychologie und Pädagogik in Anlehnung an Struktur und Niveau der deutschsprachigen Universitäten aufbauen, gleichzeitig aber sollte er dafür Sorge tragen, dass innerhalb von 10 Jahren die philosophische Lehre und Forschung in estnischer Sprache geleistet werden könne. Beiden Aufgabe kam SchmiedKowarzik zusammen mit Mitarbeitern nach, sodass die von ihm vertretene bewusstseinsanalytisch-geisteswissenschaftliche Philosophie auch nach sei-

$\mathrm{Zu}$ Leben und Werk von Walther Schmied-Kowarzik siehe den Gedenkband zum 100. Geburtsjahr (Schmied-Kowarzik 1985b).

4 Völlig überarbeitete 2. Aufl. des 1. Teils (Schmied-Kowarzik 1928). 
ner Wegberufung 1927 an die Pädagogische Akademie in Frankfurt am Main durch seine estnischen Nachfolger auf den Professuren für Philosophie, Psychologie und Pädagogik fortgeführt werden konnte (siehe von Rauch 1985 sowie Adams 1985).

Als Vorstand des Seminars für Philosophie, Psychologie und Pädagogik hatte Schmied-Kowarzik zusammen mit dem Privatdozenten Walter Freymann, sowie den Mitarbeitern Konstantin Ramul und Alfred Koort, die vor allem die Psychologie und Pädagogik betreuten, das gesamte Lehrgebiet neu aufzubauen. In den 13 Semestern, die Walther Schmied-Kowarzik in Tartu lehrte, trugt er neben einem Zyklus zur Geschichte der Philosophie einführende Grundlegungen zur Logik, Erkenntnistheorie, Kulturphilosophie, Ethik und Ästhetik vor mit begleitenden Seminare zu den großen Denkern der Neuzeit und zur Gegenwartsphilosophie. Neben eigenen UniversitätsFestvorträgen zu Dante (1921), Kant (1924) und Meister Eckhart (1926) betreute er auch die öffentlichen Vortragsreihen „Geschichte der deutschen Philosophie im 19. Jahrhundert und in der Gegenwart“ (1923/24) sowie „Die großen Mystiker aller Völker und Zeiten“ (1925/26). Sein eigener Forschungsschwerpunkt lag in diesen Jahren in der Kulturphilosophie, zu der er einige Arbeiten publizierte: so vor allem die Studie Die Objektivation des Geistigen zuerst 1927 in der Reihe Acta et Commentationes Universitatis Tartuensis (Dorpatensis). ${ }^{5}$ Nach seiner Wegberufung nach Frankfurt am Main 1927 konnten seine estnischen Mitarbeiter und Schüler - Konstantin Ramul, Walter Freymann, Alfred Koort - seine Nachfolge antreten und die bewusstseinsanalytisch-geisteswissenschaftliche Traditionslinie bis zur Okkupation Estlands durch die Sowjetunion 1940 weiter fortführen. Danach wurde diese philosophische Richtung in Tartu liquidiert - oder wie wir heute so schön Neudeutsch sagt: das Philosophische Seminar wurde „abgewickelt."

Neben dem Aufbau des philosophischen Seminars an der Universität Tartu nimmt sich Walther Schmied-Kowarzik auch der Belange der deutschsprachigen Minderheit in Estland an (vgl. auch Schmied-Kowarzik 1997). So gibt er 1923-1927 unter dem Pseudonym Dr. W. Albrecht den Estländischdeutschen Kalender heraus, das literarische Jahrbuch der Deutschbalten Estlands, das neben der deutschsprachigen Zeitung Revaler Bote eines der wichtigsten Organe der deutschsprachigen Kultur Estlands in den 2oer Jahren darstellt. Im Estländisch-deutschen Kalender veröffentlicht Walther SchmiedKowarzik einige Abhandlungen zu Fragen nationaler Kulturarbeit und erreicht darüber hinaus, dass neben deutschen auch namhafte estnische, lettische, finnische und ungarische Autoren an der Debatte um national-kultu-

5 Als eigenes Buch erschienen (Schmied-Kowarzik 1927a), siehe auch (Schmied-Kowarzik 1924b) sowie (Schmied-Kowarzik 1925). 
relle Fragen teilnehmen. Seine eigenen kulturpolitischen Artikel aus dieser Zeit sind deshalb vielschichtiger als seine kulturpolitischen Arbeiten aus den Weltkriegsjahren (Schmied-Kowarzik 1917; Schmied-Kowarzik 1918a), da er hier einerseits die deutsch-nationale Kulturpolitik aus der Sicht einer um ihre kulturelle Existenz kämpfenden deutschen Minderheit reflektiert und andererseits gleichzeitig auf die Probleme nationaler Identitätsfindung des kleinen estnischen Volkes unmittelbar an den Grenzen des russisch-sowjetischen Reiches eingeht (siehe Albrecht 1923).

Nach seiner Wegberufung aus Tartu lehrte Walther Schmied-Kowarzik an der Pädagogischen Akademie in Frankfurt am Main von 1927 bis zu deren endgültigen Auflösung 1933 Philosophie und Psychologie mit einem besonderen Schwerpunkt auf die Grundfragen der Ethik (1932). ${ }^{6}$ Durch die Schließung der Pädagogischen Akademien in Preußen 1933 musste Walter Schmied-Kowarzik seine akademische Karriere nochmals von vorne beginnen, was zur finanziellen Absicherung nicht ohne gleichzeitigen Eintritt in den Schuldienst ging. Seit 1933 lehrte er als Privatdozent Philosophie an der Universität Gießen und unterrichtete gleichzeitig an einem Gymnasium in Friedberg Geschichte und Geographie, um so für den Unterhalt seiner Familie aufkommen zu können. 1939 wurde er in den Schuldienst nach Wien versetzt und erhielt die venia als apl. Prof. an seiner Heimatuniversität Wien zu lehren. Nach dem Zweiten Weltkrieg lebte Walther Schmied-Kowarzik mit seiner Familie in Bayern, zuletzt in Regensburg, wo er an seinem letzten Buch Frühe Sinnbilder des Kosmos. Gotteserlebnis und Welterkenntnis in der Mythologie schrieb, das 1974 posthum herauskam (Schmied-Kowarzik 1974). Walther Schmied-Kowarzik verstarb am 24. Juli 1958 in seinem Vaterhaus in Mödling bei Wien - drei Tage bevor das ihm von der Philosophischen Fakultät der Universität Wien für seine wissenschaftlichen Verdienste verliehene Goldene Doktordiplom eintraf (Schmied-Kowarzik 1985a).

\section{Der philosophische Ansatz}

Ausgangspunkt des Philosophierens von Walther Schmied-Kowarzik ist die transzendentalphilosophische Rückwendung des denkenden Subjekts auf die Formen seines Bewusstseins. In der Selbstgewissheit unseres Bewusstseins gründet nicht nur all unser Erkennen, Fühlen und Streben, sondern sie ist auch Bedingung der Möglichkeit dafür, dass wir uns der Formen unseres Bewusstseins analytisch, d.h. in denkendem Selbstbezug, innewerden können. Dieser Aufgabe einer transzendentalphilosophischen Bewusstseinsanalyse stellt sich Walther Schmied-Kowarzik - anknüpfend an die österreichische phänomenologische Schule Franz Brentanos und seiner

\footnotetext{
${ }^{6}$ Siehe auch (Schmied-Kowarzik 1933).
} 
Schüler Alexius Meinong, Christian Ehrenfels und Alois Höfler - in seinem erkenntnistheoretischen Hauptwerk Umriß einer neuen analytischen Psychologie (1912/1928). Die selbstreflexive Bewusstseinsanalyse wird darin ganz entschieden abgegrenzt von allen Formen und Methoden empirischer Psychologie, also auch von jeglicher Psychologie der inneren Selbstbeobachtung und Selbsterfahrung, vielmehr versucht sie streng philosophisch - und das meint eben "analytisch“ - vorzugehen.

Die Parallelen zu Edmund Husserls fast gleichzeitig erschienenen Ideen zu einer reinen Phänomenologie und phänomenologischen Philosophie (1913) sind unverkennbar. Walther Schmied-Kowarzik hat dies auch in der zweiten Auflage seines Buches Umriß einer analytischen Psychologie (1928) ausdrücklich thematisiert. So unübersehbar die Verwandtschaft zu Edmund Husserls Phänomenologie - aber auch zu Alexius v. Meinongs Untersuchungen zur Gegenstandstheorie und Psychologie (1904) - ist, sowohl terminologisch als auch methodologisch geht Walther Schmied-Kowarzik mit seiner Analytischen Psychologie eigene Wege (Schmied-Kowarzik 1928).

\subsection{Die Analyse des subjektiven Geistes}

Das Programm der Analytischen Psychologie ist eine streng philosophische Analyse der Bewusstseinsformen oder der Formen des subjektiven Geistes, wobei es nicht nur um die Formen des Erkennens geht. Die Analyse setzt tiefer an: Ausgehend von der Selbstgewissheit der Einheit des Bewusstseins im Ich und Jetzt, differenziert Schmied-Kowarzik die „Dreiheit der Wirklichkeitserlebnisse: Empfindung, Gefühl und Strebung, "schreitet von da erst zu dem allen Wahrnehmen, Vorstellen und Erkennen zugrunde liegenden "Gegensatz von Subjekt und Objekt" fort, um schließlich den Unterschied von Denken in zergliedernden Urteilen und Intuition als ganzheitlicher Gestalterfassung insbesondere im Hinblick auf das ästhetische Erleben herauszuarbeiten.

Eine zentrale Rolle spielt zunächst die Analyse der Raum- und Zeitanschauung, die Walther Schmied-Kowarzik bereits in seiner umfangreichen Dissertation Zeit und Raum. Eine psychologische und transzendentalphilosophische Untersuchung (1908) eingehend analysiert hatte. Es ist hierbei anzumerken, dass der erste „psychologische Teil“ der Dissertation bereits ausdrücklich nicht empirisch verfährt, sondern — wenn auch noch unentfaltet - eine "transzendental-phänomenologische“ Analyse der Bewusstseinsinhalte der Zeit und des Raumes darstellt, während der zweite "transzendental-philosophische Teil“ eine metaphysische oder fundamentalphilospohische Erörterung "transsubjektiver," "transempirischer" und "metakosmischer Probleme" des Raumes und der Zeit vorlegt.

Die Dissertation Zeit und Raum von Walther Schmied-Kowarzik kann 
also auch hier - parallel zu Husserls Vorlesungen zur gleichen Zeit (Husserl 1966; Husserl 1973) - als einer der ersten Versuche einer Phänomenologie des Raumes und der Zeit gelten, wobei er stärker als Husserl einerseits auf Kant zurückgeht, den er allerdings bewusstseinsanalytisch über das Kantsche Selbstverständnis hinaus fortzuentwickeln versucht, und andererseits in den fundamentalphilosophischen Partien sich auf Leibniz zurückbezieht. Allerdings konnte diese Dissertation von 1908 nicht in die weitere Diskussion eingreifen, da sie zunächst unveröffentlicht blieb. 1910 veröffentlichte Walther Schmied-Kowarzik überarbeitet und erweitert nur den ersten Teil unter dem Titel Raumanschauung und Zeitanschauung (Schmied-Kowarzik 1910).

Schon diese Teilveröffentlichung löste zwischen den diversen Schulen der Phänomenologie (Husserl), der geisteswissenschaftlichen Philosophie (Dilthey) und der Gegenstandstheorie (Meinong) eine lebhafte Diskussion aus; wobei man Schmied-Kowarzik, insbesondere von der stärker logischempiristisch orientierten Grazer Schule der Gegenstandstheorie her, vor allem seinen Rückgriff auf Kant ankreidete, da dieser doch schon längst von den empirischen Untersuchungen von Carl Stumpf widerlegt sei (vgl. Krug 1915). Auch Rudolf Carnap bezieht sich in seiner Dissertation Der Raum. Ein Beitrag zur Wissenschaftslehre (1922) nur auf diese Veröffentlichung des psychologisch-phänomenologischen Teils der Dissertation von SchmiedKowarzik aus dem Jahre 1910. Dabei ist in dieser Teilveröffentlichung nicht nur der ganze umfangreiche transzendentalphilosophische Teil ausgeklammert. So blieben die Originalfassung der Dissertation Zeit und Raum von 1908 und insbesondere deren transzendentalphilosophische Erörterungen aus dem weiteren wissenschaftlichen Diskurs ausgeschlossen (SchmiedKowarzik 1908). Erst in einer seiner letzten philosophischen Abhandlungen „Das unendliche Sein und das endlich Seiende“ (1951) hat Walther SchmiedKowarzik in einer Auseinandersetzung mit Martin Heideggers Studie Was ist Metaphysik? (1929/49) erläuternd nochmals auf den noch unveröffentlichten transzendentalphilosophischen Teil seiner Dissertation Raum und Zeit zurückgegriffen.

Für unseren Zusammenhang reicht es aus, darauf hinzuweisen, dass der Ausgangspunkt des Philosophierens von Walther Schmied-Kowarzik in der transzendentalphilosophischen Analyse des subjektiven Geistes und seiner Formen liegt. Die Grundlage ist dabei der das „Bewußtsein als Ganzes“ tragende Selbstbezug von Ich und Jetzt, die Selbstgewissheit des Bewusstseins und ihre Selbstpräsenz. ${ }^{7}$ Von diesem Fundament her lassen sich sodann die Wirklichkeitserlebnisse in ihrer Dreiheit: Empfindungen, Gefühle und Strebung analysieren und differenzieren. Wobei es Schmied-Kowarzik über

\footnotetext{
7 Hier bestehen starke Bezüge zu (Hönigswald 1925).
} 
Kants transzendentalphilosophische Analyse der reinen Anschauungsformen von Zeit und Raum und der Kategorien des verständigen Erkennens hinaus darum geht, zu einer zergliedernden und beschreibenden Analyse der Formen der Bewusstseinsinhalte zu kommen. In dieser Weise untersucht er beispielsweise die „Gesichtsempfindungen, " nach den sie strukturierenden Momenten der Farbwahrnehmung oder die der "Gehörempfindungen“ nach den sie strukturierenden Momenten von Tonfolgen, um hierdurch aufzuzeigen, dass den menschlichen Sinnen keineswegs ein Chaos von Empfindungseindrücken zugrunde liegt, sondern, dass den Empfindungen selbst bereits vor aller kategorialen Urteilsbildung eine inhaltliche Strukturierung innewohnt. Erst auf dieser Basis der inneren Strukturiertheit der Empfindungen, Gefühle und Strebungen kann dann zu einer Thematisierung der reflexiven Trennung von Subjekt- und Objekterkenntnis und zur Differenzierung von bestimmendem Denken und intuitivem Ganzheitserfassen fortgeschritten werden (Schmied-Kowarzik 1912, 20).

Für alle so ansetzenden transzendental-phänomenologischen Positionen stellt sich - da sie ja bei der Selbstgewissheit des Bewusstseins, des Subjekts beginnen - das grundlegende Problem von Erkennen und Verstehen des Anderen. Mit Erkennen und Verstehen ist hier noch nicht die Unterscheidung wissenschaftlicher Vergegenständlichungsmethoden gemeint, sondern die diesen vorausliegende Ermöglichung der Anerkenntnis des Anderen als eines außer der Selbstgewissheit des je eigenen Bewusstseins existierenden eigenständigen anderen Bewusstseins und Subjekts und der Verständigung mit ihm. Bereits Wilhelm Diltheys späte Arbeiten kreisen um dieses Problem des Verstehens von Fremdpsychischem als Grundlage aller Geisteswissenschaften (Dilthey 1970), und ebenso ringt Edmund Husserl in seinen späteren Entwürfen zur Phänomenologie um das Problem der Intersubjektivität (Husserl 1977).

\subsection{Die Analyse des objektiven Geistes}

Im Anschluss an die geisteswissenschaftliche Verstehenstheorie von Dilthey und den phänomenologischen Ansatz von Husserl hatte Hans Freyer mit seiner Theorie des objektiven Geistes (1923) den Versuch unternommen, eine alle Geschichts- und Sozialwissenschaften fundierende Kulturphilosophie aufzubauen. Wobei er über eine Differenzierung der Formen des objektiven bzw. objektivierten Geistes zu einem wissenschaftlichen Verstehen und phänomenologischen Beschreiben geschichtlich vergangener und gegenwärtiger Kultursysteme zu kommen versucht. Hieran knüpft Walther SchmiedKowarzik in seiner Studie Die Objektivation des Geistigen (1927a) kritisch an. Wobei er allerdings bereits am Kernstück der Untersuchungen von Freyer, nämlich den „objektiven Geist und seine Formen, “ grundlegende analyti- 
sche Präzisierungen und Abgrenzungen vornimmt, die zu einer wesentlich modifizierten und erweiterten Systematik einer umfassenden Kulturphilosophie führen. Die Hauptlinien dieser Differenzierungen seien hier im folgenden unter Einbeziehung von Schmied-Kowarziks weiterer kulturphilosophischer Abhandlungen knapp umrissen (Schmied-Kowarzik 1924b). ${ }^{8}$

Vorweg sei noch darauf hingewiesen, dass sich das Problem des Erkennens und Verstehens des Anderen für Walther Schmied-Kowarzik nicht erst mit der Anerkenntnis und der Verständigung mit dem anderen Subjekt stellt, sondern bereits gegenüber dem Sein alles Seienden auftritt, wie er dies bereits im letzten „metaphysischen“ Teil seiner Dissertation Zeit und Raum dargelegt hat. Mit Rückgriff auf Kants Kritiken und die Monadologie von Leibniz macht Schmied-Kowarzik deutlich, dass das naturwissenschaftliche Erklären immer nur die endlich Seienden in ihren kausalen Beziehungen in Raum und Zeit zu bestimmen vermag, nicht aber das ihnen je zugrunde liegende Wirklichsein. Allein über unser eigenes monadisches Wirklichsein haben wir einen zeit- und raumlosen unmittelbaren Bezug zum unendlichen Sein der „Urmonade.“ Anderes Wirklichsein wird uns dagegen nicht unmittelbar, sondern nur als Objektivation des Seins erfahrbar. Hierauf ist bei Behandlung des Gottesproblems nochmals zurückzukommen.

Deutlicher wird die Problemstellung in Bezug auf das Lebendige. Wir erfassen anderes Leben nicht erst - oder gerade nicht - aus seiner wissenschaftlichen Vergegenständlichung, sondern der Erfahrung des Lebendigen liegt vielmehr unser ursprüngliches Erfassen von Lebendigem aus der Selbstgewissheit eigenen Lebendigseins und ein Verstehen der Lebensäußerungen anderer, d.h. der Objektivation des Lebens, zugrunde. SchmiedKowarzik deutet dieses Problem nur mit einigen Hinweisen in einer Tabelle der "Formen der Objektivationen des Lebens“ an (Schmied-Kowarzik 1927a, 17), ein Themenfeld, das dann Helmuth Plessner in seinem wichtigen Buch Die Stufen des Organischen und der Mensch (1928) streng methodisch als phänomenologische Hermeneutik des Lebens umfassend erschlossen hat.

Aus dem Bereich der „Objektivationen des Lebendigen“ ist sodann die „Objektivation des Seelischen“ herauszuheben, d.h. das Erfassen des anderen Subjekts aus seinen psychischen Äußerungen - z.B. „Weinen, Wimmern, Lachen, Jauchzen“ (Schmied-Kowarzik 1927a, 8). Das Fundament zu einer Theorie des Verstehens von Fremdseelischem hat insbesondere Wilhelm Dilthey mit seiner Triade: Erlebnis - Ausdruck - Verstehen gelegt (Dilthey 1970) und Eduard Spranger hat sie in seiner Abhandlung Zur Theorie des Verstehens und der geisteswissenschaftlichen Psychologie (1918) grundlegend weiterentwickelt (Vgl. Schmied-Kowarzik 1927b).

Nun erst sind wir soweit, die „Objektivationen des Geistigen“ richtig ins

${ }^{8}$ Sowie (Schmied-Kowarzik 1930). 
Auge fassen zu können, wobei es ein Missverständnis wäre, die vorausgehenden Differenzierungen als Stufen oder Seinsschichten im Sinne von Max Scheler oder Nicolai Hartmann zu verstehen, vielmehr handelt es sich um analytische Scheidungen von Problemebenen. Von einer Objektivation des Geistigen kann überhaupt nur gesprochen werden, wo Manifestationen eines Sinngehaltes vorliegen, und das bedeutet, dass ihnen immer - selbst wenn es sich um individuelle Sinnäußerungen einzelner Subjekte handelt mitmenschliche Bezüge im kulturellen Kontext zugrunde liegen. In der Objektivation des Geistigen wird kulturelles Menschsein allererst fassbar, und die Frage nach den Formen des objektiven Geistes ist im letzten die Frage nach den Grundformen, in denen sich unser kulturelles Leben zu äußern vermag.

Hans Freyer benennt in seinem Buch fünf Formen des objektiven, bzw. objektivierten Geistes, in denen sich alles kulturelle Leben vergegenständlicht: Gerät, Gebilde, Zeichen, Sozialform und Bildung. Walther SchmiedKowarzik zeigt nun auf, dass diese fünf Formen nicht nur - wie bereits Jonas Cohn sagte - „rhapsodisch“ aufgegriffen sind, sondern dass in ihnen auch völlig Heterogenes aus verschiedenen Problemebenen nebeneinander gereiht wird. Mit gewissen Modifikationen lassen sich die ersten drei als Formen des objektiven Geistes ausweisen, während Bildung und Sozialform Formen des subjektiven, bzw. ideellen Geistes darstellen.

Unter Gerät sind nicht nur Gebrauchsgegenstände wie Krug und Korb, Werkzeug und Waffe zu verstehen, sondern auch alle anderen einen Zweck erfüllende Vergegenständlichungen, wie beispielsweise Kleidungsstücke, Häuser und Verkehrsmittel. Unter Gebilde versteht Freyer das völlig zweckfreie, auf nichts außer sich verweisende, nur in sich selbst ruhende Kunstprodukt und Kunstwerk. Abgesehen davon, dass die Bezeichnung Gebilde, die eigentlich auf alles Objektivierte zutrifft, recht unglücklich gewählt ist, lassen sich nicht alle Kunstprodukte und Kunstwerke als völlig in sich ruhend, auf nichts außer sich verweisend begreifen. Im Rückgriff auf seine vorausgehenden Abhandlungen zur Ästhetik (siehe insbesondere SchmiedKowarzik 1924a sowie Schmied-Kowarzik 1925) betont Schmied-Kowarzik, dass man hier noch die Form des Abbilds hinzufügen müsse. Denn die Mammutzeichnung an der Höhlenwand oder das Portrait oder die figürliche Plastik ruhen zwar als Kunstwerke in sich, verweisen aber zugleich über sich hinaus auf anderes. In der Kennzeichnung der Form des Zeichens stimmen Freyer und Schmied-Kowarzik weitgehend wieder überein. Unter das Zeichen als Form objektivierten Geistes fällt in erster Linie die Sprache, die Schrift, die Zahl und die Formel, aber auch das Signal und das Symbol; also alles, was als Sinnverweis für anderes steht.

Von entscheidender Bedeutung ist jedoch Schmied-Kowarziks Abgren- 
zung von Freyers Kategorien der Bildung und der Sozialform. Freyer zählt die Bildung zu den Formen objektivierten Geistes, da an Denken und Handeln des gebildeten Individuums eine "formbestimmte Regelhaftigkeit" sichtbar wird. Demgegenüber erklärt Schmied-Kowarzik entschieden: „Bildung gehört ...zum beharrenden (d.h. hier geformten) subjektiven Geist und ist so das Gegenteil des objektiven Geistes" (Schmied-Kowarzik 1927a, 32). Es ist klar, um was es Schmied-Kowarzik in diesem Streit geht. Bildung ist gerade nicht etwas Vergegenständlichtes und auch nicht eine Objektivation als Äußerungsform, sondern sie ist lebendiges Formwerden und Formsein des subjektiven Geistes, ja ganz im Gegenteil, sie ist das eigentliche produktive Zentrum aller kulturellen Objektivationen. Allerdings kann der Prozess der Formung des subjektiven Geistes nur über die Aneignung des objektiven Geistes, d.h. nur vermittels der symbolisierenden, gestaltenden und zweckbezogenen Formen des objektiven Geistes erfolgen. Bildung ,ist Übertragung geistiger Werte von einem Menschen zum anderen, ist Erweckung geistigen Lebens im anderen mit Hilfe des objektiven Geistes" (SchmiedKowarzik 1927a, 34). Diese Argumentation hat Richard Meister später aufgegriffen und in seinen grundlegenden Arbeiten zur kulturphilosophischen Pädagogik weiter ausgebaut (vgl. Meister 1947 siehe auch Meister 1943).

Für das Gesamtkonzept einer Kulturphilosophie, wie sie SchmiedKowarzik vorschwebt, ist es also von entscheidender Wichtigkeit, dass Bildung nicht als objektivierte Form verstanden wird, sondern als Formwerden und Formsein des subjektiven Geistes, vermittelt über den objektiven Geist, dessen produktives Zentrum jedoch immer der subjektive Geist bleibt. Die Objektivation des Geistigen ist die notwendige Äußerungsform über die allein Subjekte sich verständigen können, aber sie ist nichts für sich, sondern sie lebt nur aus den Subjekten, die sich in diesen Formen äußern und das Geäußerte wieder aneignen. Von daher besteht Schmied-Kowarzik auch darauf, dass insbesondere der pädagogische Bildungsprozess nicht als ein bloßer Prägungsprozess durch Objektiviertes gesehen werden kann, da es gerade hier bei der Formwerdung des subjektiven Geistes auf eine möglichst dichte Begegnung der Subjekte über die unmittelbare Objektivation ankommt (Schmied-Kowarzik 1927a, 34).

Auch mit der Kategorie der Sozialform verlässt Freyer - wie SchmiedKowarzik herausarbeitet - die Analyseebene der Formen des objektiven Geistes, bzw. verfestigten Wirkzusammenhänge des sozialen Lebens. Zur Sozialform zählt Freyer neben der Sitte, die Familie, das Geschlecht, die Dorfgemeinschaft, das Volk, den Staat und den Verein. "Auch hier" - so schreibt Schmied-Kowarzik - „ist er von seiner Aufgabe der Beschreibung der Objektivationsformen in die Aufgabe der Charakteristik der Kultursysteme, der Sinngehalte hinübergeglitten" (Schmied-Kowarzik 1927a, 28). 
Denn ganz offensichtlich handelt es sich hier nicht um Objektivationsformen, sondern um Vergesellschaftungsformen oder Organisationsformen in Gemeinschaft handelnder Subjekte. Natürlich kommen diese Vergesellschaftsformen nicht ohne symbolisierende, gestaltende und zweckbezogene Objektivationen aus, und von außen betrachtet, verschmilzt beides ineinander, trotzdem müssen wir hier darauf achten, dass wir das sich organisierende gemeinsame Handeln von Menschen in einer bestimmten Kultur nicht zu einer Form objektivierten Geistes soziologisierend vergegenständlichen.

Bereits in einer vorausgehenden Auseinandersetzung mit Wilhelm Wundts Völkerpsychologie (1921) und im Anschluss an Friedrich Jodls Culturgeschichtsschreibung (1878) hat Schmied-Kowarzik sich mit den besonderen Aufgaben der Erforschung des Gemeinschaftslebens als einer lebendigen Wechselwirkung der vergesellschafteten Subjekte befasst und dabei ausdrücklich herausgearbeitet, dass man das Gemeinschaftsleben nicht als Kollektivobjekt als für sich abgeschlossene Vergegenständlichung behandeln darf (Schmied-Kowarzik 1924b). In der Wechselwirkung der Mitglieder einer Gemeinschaft bzw. der „Wechselwirkung zwischen dem Einzelnen und der Gemeinschaft“ steckt eine eigentümliche „teleologisch, “auf das gemeinsame Zusammenleben bezogene Struktur, die nicht nur in unterschiedlicher Weise die Gemeinschaft in der Familie, dem Dorf, dem Volk etc. prägt, sondern auch die gesamte kulturelle Menschheitsgeschichte zielbestimmt; ihrer Aufhellung dient die kulturphilosophische „Sozialphilosophie,“ deren letzter Problemhorizont die „Geschichtsphilosophie“ ist.

Um die dritte und eigentlich zentrale Ebene der Kulturphilosophie, die Analyse der Kultursysteme, noch deutlicher hervorzuheben, muss auf eine weitere Abgrenzung eingegangen werden. Richard Meister, der in vielen Punkten Schmied-Kowarzik folgt, hat zu den besprochenen Formen der Objektivation des Geistigen noch als weitere (vierte) Kategorie die Norm hinzugefügt, und Friedrich Kainz übernimmt diese Ergänzung (Kainz 1977). Nun hat jedoch Walther Schmied-Kowarzik in seiner Arbeit Objektivation des Geistigen bereits vorweg begründet, warum für ihn die Norm keine Form des objektiven Geistes sein kann: „Das geistige Leben als solches ist Erlebnis, Akt, fließendes Seelenleben, und es ist gerichtet auf einen Sinngehalt. Dieser Sinngehalt ... ist selbst nicht eine seelische Wirklichkeit, ein Akt des Erlebens, ist (aber) auch keine körperliche Wirklichkeit, überhaupt keine Wirklichkeit, sondern gehört einer idealen Sphäre, dem sogenannten dritten Reich an, dem Gültigkeit, aber nicht Dasein, Existenz ... zugeschrieben werden muss. Das geistige Leben schafft dann in der physischen Welt Gebilde als Träger und Mittler „gegenständlichen“ Sinngehalts (symbolische Handlungen, Kunstwerke, Geräte usf.)“ (Schmied-Kowarzik 1927a, 3). Walther Schmied-Kowarzik geht also von einer Dreigliedrigkeit von subjekti- 
vem, objektivem und ideellem Geist aus, wobei er selber ausdrücklich auf die Differenz zur Dreigliederung bei Hegel hinweist.

\subsection{Die Analyse des ideellen Geistes}

Der Ideelle Geist - und dazu gehören genauso die Normen des Rechts und der Sittlichkeit wie der Wahrheit und Schönheit - wird als solcher nicht objektiv, weder im Psychischen noch im Physischen. ${ }^{9}$ Wohl können sich menschliche Handlungen an ihnen als „regulativen Ideen" (Kant) orientieren, und so kommt auch etwas von ihnen nicht nur im Handeln der Menschen zum Vorschein, sondern die ganze Kulturgeschichte lässt sich am Grad ihrer differenzierteren Verwirklichung bemessen, trotzdem geht das Ideelle - beispielsweise das Sittengesetz - wie immer es im Laufe der Geschichte ausformuliert werden mag, niemals in dem auf, was von ihm im sittlichen Handeln der Menschen einer Kultur manifest wird, sondern - wie schon Platon von der Idee sagte — gibt es hier immer nur eine „Teilhabe“ des Handelns am ideellen Geist, der durch alle kulturellen Objektivationen hindurch immer nur indirekt zum Vorschein kommt.

Von hier her sei nochmals die Frage aufgenommen, was denn die Sphäre des ideellen Geistes sei, die - wie Schmied-Kowarzik betont - keine bloße Gegebenheit des subjektiven Geistes ist und deren „Objektivierungen ...nicht an außerleibliche Produkte gebunden zu sein " brauchen (SchmiedKowarzik 1927a, 12). Mit jenen ideellen Sinngehalten des Rechts, der Sittlichkeit, aber auch der Wahrheit, der Schönheit und des Religiösen gehen teleologische, richtungsweisende Momente in all unser mitmenschliches Handeln ein - wie Schmied-Kowarzik insbesondere in seiner Ethik (1932) näher ausführt. Aber was sind diese „regulativen Ideen,“ da sie doch keinesfalls als "metaphysische Wesen“ oder „selbständige Substanzen“ begriffen werden können?

Es sei dieses Problem hier nur noch beispielhaft an dem Fundamentalproblem des Religiösen angedeutet, das wie ein roter Faden das gesamte Philosophieren Schmied-Kowarziks von den Schlusskapiteln seiner Dissertation Zeit und Raum (1908) an bis zur Abhandlung „Das unendliche Sein und das endliche Seiende" (1951), sowie von der religionsphilosophischen Grundlegung "Gotteserlebnis und Welterkenntnis" (1918) bis zum letzten Buch Frühe Sinnbilder des Kosmos (1958) durchzieht.

Sicherlich sind unsere religiösen Vorstellungen in all ihrem bildlichen und gedanklichen Bestimmungen Objektivationen unseres Geistes, dies ist nicht erst eine Entdeckung Ludwig Feuerbachs, sondern gut zweieinhalb

9 Siehe hierzu die Auseinandersetzung von Schmied-Kowarzik mit Ernst Cassirer abgedruckt in (Cassirer 1985). 
Jahrtausende vorher hat diese Einsicht bereits Xenophanes ausgesprochen. Aber dies ist nur die halbe Wahrheit - wie Schmied-Kowarzik in „Gotteserlebnis und Weltkenntnis" in betontem Anschluss an Friedrich Schleiermacher ausführt -, denn in der existentiellen Einsicht unserer Endlichkeit tritt zugleich das „Gefühl schlechthinniger Abhängigkeit“ vom Universum auf (Schleiermacher); wir sind also "notwendig gottsetzendes Bewusstsein“ (F.W.J. Schelling), da sich der menschliche Geist nicht aus sich selbst heraus zu konstituieren vermag.

Nun haben jedoch einige diese Verankerung der Glaubensgewissheit im Gefühl lediglich als ein „metaphysisches Bedürfnis“ in uns (Arthur Schopenhauer) gefasst, nicht aber als einen wirklichen Bezug zum Sein oder Gott verstanden. Dieses Gefühl allein festgehalten und der wissenschaftlichen Erkenntnis von der Welt ohne Gott gegenübergestellt, führt zum radikalen Als-ob-Standpunkt Hans Vaihingers (1911). „Die Philosophie des Als-ob verneint den Wahrheitsgehalt der Glaubensvorstellungen und das Dasein des Göttlichen“" (Schmied-Kowarzik 1918b, 139). Sie macht die Gottesvorstellung zu einem "Scheingebilde," ohne das wir zwar nicht auskommen, das aber doch keine „Daseinsbedeutung" und keinen „Wahrheitsgehalt" für uns hat; dies führt letztlich zu einer Bedeutungslosigkeit des Religiösen.

Diesem radikalen Als-ob-Standpunkt setzt nun Walther SchmiedKowarzik einen - wie er im Anschluss an Friedrich Jodl sagt - „eingeschränkten Als-ob-Standpunkt", aus kritischer Metaphysik begründet, entgegen, indem er zunächst in negativer Erkenntniskritik die Unbegründbarkeit der wissenschaftlichen Erkenntnisweise in ihrem Absolutheitsanspruch dartut. Niemals vermag die wissenschaftliche Erkenntnis das unendliche, ewige, absolute Sein zu erreichen, das doch gleichwohl allem endlich Seienden zugrundeliegen muss. Gerade an unserer Raum- und noch entschiedener an unserer Zeiterfahrung hat Schmied-Kowarzik seit seiner Dissertation deutlich zu machen versucht, dass wir hier in eine für das wissenschaftliche Erkennen unlösbare Aporie getrieben werden, denn dieses kann nur Endliches in Zeit und Raum bestimmen, bedarf aber gleichwohl zur Bestimmung von Endlichem der absoluten Gegenposition, die sich ihrem Zugriff aber prinzipiell entzieht.

Ganz anders aber steht es mit dem „Gefühl“ unseres existentiellen Daseins im „Universum“(Schleiermacher). „Zur Anerkenntnis des Seiens eines Absoluten, Unendlichen gelangen wir durch Vertiefung in den Gedanken des Endlichen" (Schmied-Kowarzik 1918b, 142), wobei dieses gerade nicht rational zergliedernd geschieden wird, sondern als ein Einbezogensein im Ganzen erlebt wird, wie dies Schleiermacher dargelegt hat: „Alles Einzelne nicht für sich, sondern als einen Teil des Ganzen, alles Beschränkte nicht in seinem Gegensatz gegen anderes, sondern als eine Darstellung des Un- 
endlichen in unser Leben aufnehmen und uns davon bewegen lassen, das ist Religion" (Schleiermacher 1958, 54). Und Schmied-Kowarzik fügt erläuternd hinzu: „Die Art der religiösen Gottesvorstellung ist also immer subjektiv, nicht ein Ebenbild der Gottheit wiedergebend, sondern ein Bild des besonderen Menschen und der ihm eigentümlichen Stellung im Ganzen des Weltall“" (Schmied-Kowarzik 1918b, 147). „Subjektiv“ meint aber hier nicht etwas Abwertendes, denn nur dem absolutgesetzten wissenschaftlichen Erkennen erscheint es so, für das religiöse Bewusstsein dagegen ist es der unmittelbare Bezug unseres existentiellen Daseins zum Sein, in dem wir Gott zu erhoffen vermögen.

Damit ist keine „doppelte Wahrheit postuliert, sondern einzig die eine Wahrheit, die durch Erkennen und Fühlen in doppelter Weise gewährleistet ist" (Schmied-Kowarzik 1918b, 148). Das wissenschaftliche Erkennen wird immer weiterfahren, uns das endlich Seiende zu differenzieren, aber es wird dabei niemals dem Sein begegnen, das es doch voraussetzen muss; wissenschaftlich lässt sich daher nie das Dasein Gottes beweisen oder ableugnen. Das existentielle Gefühl unseres Endlichseins dagegen erlebt sich unmittelbar aus dem unendlichen Sein und vermag alles Seiende als Objektivationen des Seins zu erfahren. Nur der Sinnbezug ist unmittelbar, seine Erfahrung als göttlicher Sinnzusammenhang und Sinnanspruch erfordert die Bildung religiöser Meditation.

Von hier her bekommen die religiösen Glaubensvorstellungen durch die Jahrhunderttausende der Menschheitsgeschichte ihren tieferen Sinn als „Objektivierungen des ideellen Geistes.“ Von der Außenperspektive des theoretischen Erkennens erscheinen sie allesamt als unbegründete und vergängliche Schöpfungen und Projektionen des menschlichen Geistes, aber für das religiöse Bewusstsein, das selbst im existentiellen Innewerden unseres endlichen Daseins im unendlichen Sein wurzelt, ist ihr Sinngehalt eine wirkliche, geschichtliche sich läuternde Offenbarwerdung gestifteter und zugleich uns aufgegebener Sinnerfüllung.

In seiner letzten religionsphilosophischen Arbeit Frühe Sinnbilder des Kosmos (1958) versucht Walther Schmied-Kowarzik, von solcher Innenperspektive her, den ideellen Sinngehalt des Mythos als ein Erlebnismoment unserer eigenen religiösen Läuterung zu erschließen, er führt damit weiter, was er vierzig Jahre vorher in den Schlusssätzen von „Gotteserlebnis und Welterkenntnis“ (1918) aussprach: „Die religiösen Vorstellungen sind aus dem Gesichtspunkt des (eingeschränkten) Als-ob zu begreifen, aber nicht als Trugbilder, sondern als Sinnbilder. Sie sind nicht wesenlose Fiktionen, nützliche Täuschungen, sondern symbolische Wahrheiten ...So wird der Glaube vom Wissen nicht aufgehoben, sondern in seinem Grundgehalt bestätigt, in seinen mannigfachen und wandelbaren Formen geläutert. Die My- 
stiker aller Bekenntnisse, die heiligstes Fühlen mit tiefsinnigen philosophischen Einsichten verbanden, sind ein lebendiges Zeugnis für diese reifste Vervollkommnung und Vergeistigung der religiösen Sinnbilder" (SchmiedKowarzik 1918b, 152).

\section{Literatur}

Adams, V. (1985). Gruß aus Tartu. Erinnerungen an meinen verehrten Lehrer, in W. Schmied-Kowarzik (hrsg.), Objektivationen des Geistigen. Beiträge zur Kulturphilosophie in Gedenken an Walther Schmied-Kowarzik (1885-1958), Reimer, Berlin.

Albrecht, W. (1923). Die nationale Gesinnung als ethische Pflicht, Estländisch-deutscher Kalender 1924, Dorpater Deutscher Volksbund, Tartu.

Cassirer, E. (1985). Symbol, Technik, Sprache. Aufsätze aus den Jahren 19271933, Meiner, Hamburg.

Dilthey, W. (1970). Der Aufbau der geschichtlichen Welt in den Geisteswissenschaften, hrsg. v. Manfred Riedel, Suhrkamp, Frankfurt am Main.

Feuerbach, L. (1903-1910). Sämtliche Werke, hrsg. v. Wilhelm Bolin und Friedrich Jodl, Frommann, Stuttgart.

Gimpl, G. (hrsg.) (1990). Unter uns gesagt. Friedrich Jodls Briefe an Wilhelm Bolin, Löcker Verlag, Wien.

Gimpl, G. (hrsg.) (1996). Ego und Alterego. Wilhelm Bolin und Friedrich Jodl im Kampf um die Aufklärung, Peter Lang, Frankfurt am Main.

Husserl, E. (1966). Zur Phänomenologie des inneren Zeitbewußtseins, Martinus Nijhoff, Den Haag.

Husserl, E. (1973). Ding und Raum. Vorlesungen 1907, Martinus Nijhoff, Den Haag.

Husserl, E. (1977). Cartesianische Meditationen, Meiner, Hamburg.

Hönigswald, R. (1925). Die Grundlagen der Denkpsychologie. Studien und Analysen, 2. Auflage, B. G. Teubner, Leipzig.

Jodl, F. (1878). Culturgeschichtsschreibung - ihre Entwickelung und ihr Problem, Engelmann, Halle.

Jodl, F. (1920). Kritik des Idealismus, Akademische Verlagsgesellschaft, Leipzig. Bearbeitet und herausgegeben von Carl Siegel und Walther SchmiedKowarzik. 
Jodl, F. (1924). Lehrbuch der Psychologie, Verlag der J.G. Gottaschen Buchhandlung, Leipzig. Herausgegeben und bearbeitet von Carl Siegel und Walther Schmied-Kowarzik.

Kainz, F. (1977). Hauptprobleme der Kulturphilosophie. Im Anschluß an die kulturphilosophischen Schriften Richard Meisters, Verlag der Österreichischen Akademie der Wissenschaften, Wien.

Krug, J. (1915). Neueres zu den Raumtheorien Kants und Stumpfs, Archiv für die gesamte Psychologie 33: 241-260.

Meister, R. (1943). Seinsformen der Kultur, Blätter für Deutsche Philosophie 17.

Meister, R. (1947). Geistige Objektivierung und Resubjektivierung: Kultur und Erziehung, Wiener Zeitschrift für Philosophie, Psychologie und Pädagogik $\mathbf{1}$

Schleiermacher, F. (1958). Über die Religion. Reden an die Gebildeten unter ihren Verächtern, Meiner, Hamburg.

Schmied-Kowarzik, Walther (1908). Zeit und Raum. Eine psychologische und transzendentalphilosophische Untersuchung, Aus dem Nachlass vollständig hrsg. v. W. Schmied-Kowarzik, Gesamthochschulbibliothek, Kassel.

Schmied-Kowarzik, Walther (1910). Raumanschauung und Zeitanschauung, Archiv für die gesamte Psychologie 1: 94-151.

Schmied-Kowarzik, Walther (1911). Intuition. Ein Beitrag zur Psychologie des ästhetischen Erlebens, J.A. Barth, Leipzig.

Schmied-Kowarzik, Walther (1912). Umriß einer neuen analytischen Psychologie und ihr Verhältnis zur empirischen Psychologie, J.A. Barth, Leipzig.

Schmied-Kowarzik, Walther (1917). Ein Weltbund des Deutschtums, Weicher, Leipzig.

Schmied-Kowarzik, Walther (1918a). Die Gesamtwissenschaft vom Deutschtum und ihre Organisation, Deutschnationale Verlagsanstalt, Leipzig.

Schmied-Kowarzik, Walther (1918b). Gotteserlebnis und Welterkenntnis, in E. Bermann (hrsg.), Festschrift für Johannes Volkelt zum 7o. Geburtstag, Beck, München.

Schmied-Kowarzik, Walther (1924a). Die Kunstform in den tektonischen Künsten, Zeitschrift für Ästhetik und Allgem. Kunstwiss. 18.

Schmied-Kowarzik, Walther (1924b). Stellung und Aufgabe von Wundts Völkerpsychologie und der Begriff des Volkes, Wilhelm Wundt - Eine Würdigung, Keyser, Erfurt.

Schmied-Kowarzik, Walther (1925). Gestaltpsychologie und Ästhetik, in 
G. della Valle (hrsg.), Atti del 5. Congresso internazionale di Filosofia 1924, Perrella, Napoli.

Schmied-Kowarzik, Walther (1927a). Die Objektivation des Geistigen. Der objektive Geist und seine Formen, J.A. Barth, Leipzig.

Schmied-Kowarzik, Walther (1927b). Diltheys und Sprangers verstehende Psychologie in ihrem Verhältnis zur erklärenden (naturwissenschaftlichen) Psychologie, Archiv für die gesamte Psychologie 58.

Schmied-Kowarzik, Walther (1928). Umriß einer neuen analytischen Psychologie. Teil I: Grundlegung einer nichtempirischen Psychologie, J. B. Barth, Leipzig. 2. umgearb. u. erweit. Aufl.

Schmied-Kowarzik, Walther (1930). Großstadt und Weltanschauung, Philosophie und Leben $\mathbf{6}$.

Schmied-Kowarzik, Walther (1932). Ethik. Mit Berücksichtigung pädagogischer Probleme, A.W. Zickfeldt, Osterwieck.

Schmied-Kowarzik, Walther (1933). Mensch und Menschentum, eine anthropologische Grundlegung, Zeitschrift für den ev. Religionsunterricht 44.

Schmied-Kowarzik, Walther (1951). Das unendliche Sein und das endliche Seiende, Wiener Zeitschrift für Philosophie, Psychologie, Pädagogik 4: 265275.

Schmied-Kowarzik, Walther (1974). Frühe Sinnbilder des Kosmos. Gotteserlebnis und Welterkenntnis in der Mythologie, A. Henn, Ratingen.

Schmied-Kowarzik, Wolfdietrich (1985a). Versuch, ein Leben zu vergegenwärtigen, in W. Schmied-Kowarzik (hrsg.), Objektivationen des Geistigen. Beiträge zur Kulturphilosophie in Gedenken an Walther Schmied-Kowarzik (1885-1958), Reimer, Berlin.

Schmied-Kowarzik, Wolfdietrich (1993). Vergessene Impulse der Wiener Philosophie um die Jahrhundertwende, in J. Nautz und R. Vahrenkamp (hrsg.), Die Wiener Jahrhundertwende. Einflüsse - Umwelt - Wirkungen, Böhlau, Wien.

Schmied-Kowarzik, Wolfdietrich (1996). Friedrich Jodls Ethik in ihrem systematischen Kontext, in G. Gimpl (hrsg.), Ego und Alterego. Wilhelm Bolin und Friedrich Jodl im Kampf um die Aufklärung, Peter Lang, Frankfurt am Main.

Schmied-Kowarzik, Wolfdietrich (1997). Kulturnationalismus - Intentionen und Gefahren, in C. Kiss, E. Kiss, J. Stagl (hrsg.), Nation und Nationalismus in wissenschaftlichen Standardwerken Österreich-Ungarns ca. 18671918, Böhlau, Wien. 
Schmied-Kowarzik, Wolfdietrich (hrsg.) (1985b). Objektivationen des Geistigen. Beiträge zur Kulturphilosophie in Gedenken an Walther SchmiedKowarzik (1885-1958), Reimer, Berlin.

Spranger, E. (1918). Zur Theorie des Verstehens und der geisteswissenschaftlichen Psychologie, in E. Bermann (hrsg.), Festschrift für Johannes Volkelt zum 70 . Geb, Beck, München.

Vaihinger, H. (1911). Die Philosophie des Als Ob, Reuther \& Reichard, Berlin. von Rauch, G. (1985). Walther Schmied-Kowarzik an der Universität Dorpat, in W. Schmied-Kowarzik (hrsg.), Objektivationen des Geistigen. Beiträge zur Kulturphilosophie in Gedenken an Walther Schmied-Kowarzik (1885-1958), Reimer, Berlin.

Wundt, W. (1921). Probleme der Völkerpsychologie, A. Kröner, Stuttgart.

On Walther Schmied-Kowarzik's Philosophy of Consciousness Analysis

Through the mediation of the Finnish philosopher Wilhelm Bolin, Walther SchmiedKowarzik (1885-1958) from Vienna/Austria would be appointed the first full professor for philosophy at the newly founded Estonian state university in Tartu in 1919. Not only was he supposed to establish the disciplines of philosophy, psychology and pedagogy based on the model of western European universities, but was also to see that within ten years these disciplines be taught in the Estonian language and that they become the fundamental building blocks for Estonian teacher training and academic culture. Walther Schmied-Kowarzik advocated a philosophy based on a (phenomenological) analysis of consciousness as it began to surface towards the beginning of the 2oth century and continues today. His interests therein were for a transcendental analysis of the forms of "subjective," "objective," and "ideational spirit." In his professorial dissertation written in Vienna, Umriß einer analytischen Psychologie (1912/1928), he laid the foundations for the differentiation of the subjective forms of human knowing, feeling, and desire through reflective thinking. Building upon this in his book Die Objektivation des Geistigen. Der objektive Geist und seine Formen (1927) published in Tartu, he developed the outlines of a philosophy of culture. After being called away in 1927 to the Pedagogical Academy in Frankfurt am Main he then turned with his book Ethik. Mit Berücksichtigung pädagogischer Probleme (1932) to the forms of the "ideational spirit," which is also the focus of his last, posthumous book Frühe Sinnbilder des Kosmos. Gotteserlebnis und Welterkenntnis in der Mythologie (1974).

Keywords: Walther Schmied-Kowarzik, philosophy of consciousness analysis (phenomenology), the forms of the subjective spirit, culture as objectification of the spiritual, custom and religion as forms of the ideational spirit 